\title{
Rational decision making; the role of physician
}

\author{
Sarath Lekamwasam ${ }^{1}$
}

Journal of the Ceylon College of Physicians, 2012, 43, 2-6

Past Presidents, Board of Trustees, Members of the Council, representatives of the Ministry of Health, professional organizations, and other health carerelated institutions, special invitees, ladies and gentlemen, at the outset, I wish to congratulate the outgoing President and the Council, for the splendid work they carried out during the last year.

It is with great pride and humility I stand before you today, as the President of Ceylon College of Physicians (CCP). I assure you that, I will sincerely and wholeheartedly dedicate my time and energy to support the College with utmost faith and care, during my office. As D Everett, the Columbian orator once said, "large streams from little fountains flow, tall oaks from little acorns grow". The CCP must have followed the same philosophy, a seed planted in fertile soil, nurtured and protected by a dedicated group of people for 45ys and now it has grown to the current dimensions. The CCP came into being on the 25th July 1967 following a discussion held in the consultant lounge of then General Hospital, Colombo. In this 45y long history, numerous individuals must have made significant contributions and must have worked tirelessly for one single cause.

The CCP is the apex organization involved in professional development, improving patient care and lobbying for good governance. It is fully committed to enhancing excellence in medical care in the country. While the College remains the foremost institution in developing management guidelines of medical disorders, it closely associates with other institutions with similar objectives, such as the Ministry of Health and the Post Graduate Institute of Medicine.

"Rational decision making; the role of physician" is the area that I have chosen to speak today. I considered physicians a different breed, from my student days. I was fortunate to learn under the physicians in the caliber of Late Dr Mrs Soma de Silva, Dr Viswarathnum and then Dr Nanda Amarasekara. They made decisions on matters related to life and

\footnotetext{
${ }^{1}$ President, Ceylon College of Physicians 2012.
}

death purely using their clinical acumen. Many times it puzzled me how they narrowed down the differential diagnosis and how they selected the most suitable investigation or treatment out of many possible options. Is it mere instinct, intuition, or experience which is predominantly a personal attribute and a difficult trait to teach someone or else does it involve a systematic process that can be identified and taught to others?

I do not intend to dwell on education principles that govern the decision making of doctors, for the reason that I am not qualified to do so. A book written by Jerome Groopman from Harvard University on "How doctors think"1 discusses this subject in great detail. Clinicians are required to make clinical decisions all the time and clinical decision making and critical thinking are somewhat similar but yet different processes. In the clinical arena, it is imperative for one to make the correct clinical decision. The information gathered from clinical history, physical examination and lab investigations should, theoretically, leads one to the correct clinical decision. But all of us know that it is not the case in many instances. When the clinician is confronted with many possibilities instead of one, differentiating one diagnosis from another is a tedious and difficult process.

Clinical decision making (CDM) is a cognitive process concerned with problem recognition, data gathering, assimilation, analysis, and evaluation, finally leading to operational decision, i.e. a decision leading to action $^{2}$. So CDM has two clear components; an assessment and evaluation. Clinicians in old days made clinical decisions entirely based on their experience. The validity of this process was questioned by Prof David Sackett, of McMaster University in Canada when he introduced the concept of Evidence. The series of articles named "The readers guide to medical literature"3 published by the Canadian Medical Association Journal in 1981 signify the beginning of a new era in clinical decision making.

Evidence-based medicine (EBM) has only one goal; i.e. to improve the health of people through decisions that will maximize their health related quality of life. Although this goal looks logical and widely acceptable for all sections of the medical profession, several years after its introduction, EBM came for 
severe criticism from some sections. This is primarily because they felt that EBM would eventually replace the clinical experience and it would occupy a supreme position in CDM.

However, we now know that EBM and clinical experience are two complimentary processes with a common goal. Evidence and experience are not the only components of the decision making. Expectations, values and circumstances of the patients or people also have a role to play ${ }^{4}$. This includes cultural and religious beliefs of patients too. The acceptability of health benefits of moderate alcohol consumption, contraceptive practices and termination of pregnancy on medical grounds varies depending on religious and moral values of patients. Similarly, patient is one of the major decision makers when it comes to invasive procedures performed as a part of either investigations or treatment.

This can get further complicated with issues such as factors related to economy of the individual and country. The decision to intervene with a pharmacological agent may depend on the willingness to pay for health by an individual, insurance company or health care agencies ${ }^{5}$. This is one of the best examples how economic factors decide who should be treated and who should not be treated. In the USA, postmenopausal women are treated with bisphosphonates only when the major osteoporotic fracture probability exceeds $20 \%$ or hip fracture probability exceeds $3 \%{ }^{6}$. This recommendation is purely based on cost effective analyses. When a similar analysis was done in the UK, using the cost of generic bisphosphonates, the treatment became cost effective at much lower (7\%) level of fracture probability ${ }^{7}$. This basically indicates that the decision to treat may depend on factors related to economy.

In day to day life clinicians make decisions, broadly, in two major areas; i.e. diagnosis and treatment. Although our wish is to get both right, experience and research tell us that we make significant errors in both areas. Generally, diagnosis is achieved by analyzing the information derived from history, examination and investigations. In old days, major contribution came from history and examination but now doctors rely, heavily, on investigations. Seldom, it is based on observations made during treatment or follow up. We should understand that information gathered from history, examination and investigations has a scientific meaning, either positive or negative, and is measurable or quantifiable. We generally consider them as Sensitivity and Specificity values. Although Sensitivity and Specificity are the commonly used concepts, predictive values which are less well known are more informative to clinicians.
Sensitivity of a lab test indicates, of people with the disease, the proportion with an abnormal result. In contrast, Positive Predictive Value (PPV) indicates, of people with an abnormal result, the proportion of people who are likely to have the disease. PPV is more useful for the clinician, as it is the abnormality detected in history, examination or investigations that you come across first and not the diagnosis. Hence it is the natural order. NPV also can be interpreted in a similar manner.

Although Sensitivity and Specificity do not, Predictive values do vary based on the disease prevalence. A positive test has a higher predictive value (higher probability of the disease) when applied to a patient population with higher disease prevalence. Similarly, a positive test would give a low probability of the disease when applied to a population with low disease prevalence ${ }^{8}$. When the clinical situation does not demand, investigations done in a haphazard manner can lead to many uncertainties. A positive test in an asymptomatic patient will call for further testing to clear the doubt and this can be a costly exercise. Furthermore, it creates a certain degree of uncertainty in the mind of the patient and in some instances patients have reacted to this type of situation adversely. The temptation to order unwarranted investigations should be resisted and discouraged. ANA, rheumatoid factor, filarial antibody, imaging techniques and ECG are the commonly misdirected investigation in this fashion. "Incidentaloma" is probably a byproduct of such investigation misuse.

Although we are unaware, the mind of the clinician work on a combination of well accepted principles in clinical epidemiology. Knowingly or unknowingly we all manage our patients according to disease probabilities. Probability simply means the certainty of an event or the truth and it varies from $100 \%$ to $0 \%$. In CDM we use probability of the suspected disease in a given patient. Above a certain cut-off level investigations are not required to decide on treatment. In some, the probability is so low that further investigations are not indicated. Only the people who are in between these two thresholds would require investigations. It is essential that such an investigation should move him either up or down the scale and should not leave him in the same position when the test result is available ${ }^{8}$. The distance they move will depend on the strength of the Likelihood Ratio (LR) attached to the test result. LR compares the probabilities of a disease before and after the test results. If the disease probability is only $5 \%$ before testing (ie only one in 20 with such clinical picture is likely to have the disease) a positive test result with LR of 10 will elevate the probability of the disease to $30 \%$ (now one in three is likely to have the disease) ${ }^{8}$.

The following table shows the LRs attached to certain physical signs ${ }^{9}$. 
Table. LRs of selected physical signs

Physical Sign and condition

1) Pulses paradoxus in predicting severe asthma

2) Rinne test detecting conductive hearing loss

3) Vocal cord paralysis for thyroid carcinoma

4) Cervical lymphadenopathy for thyroid carcinoma

5) Eyelid retraction for hyperthyroidism

6) Lid lag for hyperthyroidism

\section{Positive Predictive} Value

22.6

16.8

45.2

13.4

31.5

17.6
Negative Predictive Value
Apart from decisions related to diagnosis, physicians make decisions related to treatment. In the era of EBM, RCTs are believed to generate the best evidence of the efficacy of interventions. The evidence pyramid which displays the hierarchical positions of various research methodologies, place RCTs at its summit while various observational studies are placed below (Figure).

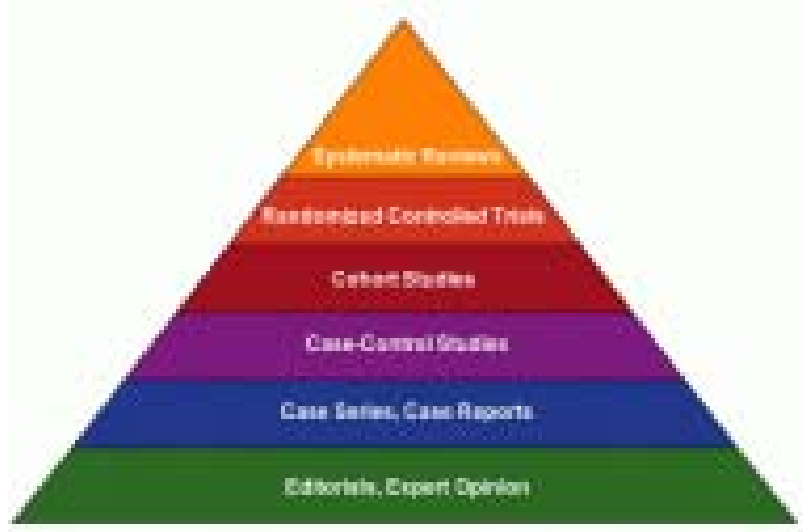

Figure. Evidence pyramid with relative positions of different research methods.

RCTs, since their introduction in the middle of the 20th century, have had a profound impact on heath care. Well conducted multicenter double-blind RCTs, unquestionably, testify for the internal validity of findings. RCTs are the bread and butter of physicians. Journal clubs, guest lectures and symposia are based on RCTs. Information gathered from RCTs are incorporated to management guidelines.

Although the hierarchy of evidence places RCTs on the top of the evidence pyramid, giving such a prominence to RTCs is unreasonable, at times. Apart for RCTs there are other ways of establishing the benefits of an intervention. Numerous pharmacological interventions or practical procedures have not gone through RCTs. They are based on physiological principles. When deficiency of a particular hormone or vitamin is associated with serious clinical outcome, choice of treatment is natural and does not require RCTs. These include insulin for type I diabetes, vitamin B12 for pernicious anemia, thyroxin for hypothyroidism and tracheostomy for upper airway obstruction.

Most RCTs now follow rigorous randomization and blinding procedures. Randomization, if properly done, ensures prognostically similar cohorts to test out the intervention. More importantly it ensures equal distribution of known and unknown confounding factors thus balancing the two groups. If a difference in the distribution of confounders is seen, it is considered to be entirely due to chance. RCTs, however, are not without limitations and imperfections. Among diverse factors that contribute to flaws in RCTs, I have chosen a few to elaborate.

When researchers take all possible precautions to improve the internal validity of studies, the external validity (generalizability of findings) tends to suffer. Always there is a trade-off between these two concepts. RCTs recruit patients with a particular condition (such as hypertension) but without a significant co-morbidity. Findings of research based on these pure subjects have limited generalizability. In real life many patients with hypertension have abnormal serum lipids, glucose or renal insufficiency.

Travers et al evaluating the eligibility of COPD patients for clinical trials found that $90 \%$ of COPD patients in the community belonged to the "excluded category" in the major trials that were used to develop management guidelines on COPD ${ }^{10}$. The same authors 
extending the survey to asthma patients found that only $4 \%$ of asthmatics in the Southampton area in the UK were eligible to enter a clinical trial based on the eligibility criteria used by the major asthma CTs on which GINA guidelines are based ${ }^{11}$. This is not an isolated phenomenon seen in respiratory medicine. It is found in all the other areas such as diabetes, heart failure, renal failure etc.

Exhaustive, often unwarranted, analysis of subgroups is another critical aspect of RCTs. If done with clear objectives, subgroup analyses generate clinically relevant information such as gender, ethnic or geographical differences of treatment efficacy. For this, subgroup analyses need to be preplanned at the beginning of the study (priori). Post-hoc multiple analyses are not only clinically meaningless, they violate principles related to statistical analysis. As probability of less than $1 / 20$ has been conventionally set to define statistical significance, multiplicity of comparisons would artificially increase the probability of finding a statistical significance with limited or no clinical relevance ${ }^{8}$.

The BARI trial which compared coronary artery by-pass graft vs coronary angioplasty in angina illustrates the dilemma created by post-hoc subgroup analyse ${ }^{12}$. Authors of this trial found no overall survival benefits, but in diabetics the mortality was nearly double in the angioplasty group compared to bypass group. As authors reported this in a convincing manner, the US National Institute of Health issued a major public heath recommendation to this effect. This was, however, a result of many exploratory subgroup analyses and the possibility of false positive finding can not be ruled out. As it was stated later in the Lancet, such a surprise subgroup result should have been a basis for further research but not influenced the national policies ${ }^{13}$. Bhandari et al in 2006, analyzing surgical trials found 27 trials to have 54 subgroup analyses with number of analyses ranging from 1 to 23 . In 91\% of these, analyses were post-hoc and had no prior hypotheses. They considered these publications to be misinformative and misleading ${ }^{14}$.

RCTs is not a reliable tool to determine the adverse effects or harms related to interventions. In principle, sample size is chosen to have the sufficient power to detect a statistically significant difference of treatment efficacy between treated and untreated groups. This calculation does not, usually, take harm into account. As a result, RCTs have the ability to recognize only common adverse effects. Less common yet clinically important adverse events are exposed only during the post-marketing surveys. According to a survey published in 1998, of the new therapies licensed as medicines in the UK between 1972 and 1994 , only one has been withdrawn for lack of efficacy but 22 for safety reasons ${ }^{15}$.
Ladies and gentlemen, in my speech, I have taken you through a series of thoughts, unrelated but in certain ways related, that occurred to me from time to time. They illustrate the difficulties encountered by clinicians in their judgmental thinking. In making clinical decisions we should take many aspects such as experience, evidence and expectation, into consideration. In the process of arriving at the diagnosis, we should use our judgment to select the most suitable test. It is a good principle to discourage performing investigations in a haphazard way and trainees should learn the process of selecting the most suitable and cost effective route to reach the diagnosis.

Furthermore, it was not my intention to downgrade the value of RCTs or question the hierarchical position given to them, currently. I only questioned some issues which potentially can compromise the value of RCTs. In addition, the intuition or life time experience of clinicians in making clinical judgment would remain intact for years to come. Evidence should never be seen as a threat to clinical experience rather it should be complementary to life time experience of the clinician. It is the totality, experience and evidence combined, that makes the best judgment.

In concluding, numerous people have made significant contributions to my career. My parents, family and teachers are the foremost. With affection, I remember all teachers in the Peradeniya Medical Faculty. Dr WAS de Silva, former Professor of Medicine, Galle encouraged me to join a university and he changed my career pathway. Prof Juliet Compston, in the Department of Medicine, Cambridge Medical School made a significant impact on my career. She got me involved in two major systematic reviews and most what I have said here originated while I was working on those two projects. I am very thankful to all my colleagues in the Faculty of Medicine, Galle especially those in the Department of Medicine. I should thank the new council of the CCP, especially two secretaries and the CCP staff. I am particularly thankful to all those present here this evening, spending your valuable time, to witness this event. Finally, my wife Vajira, and two children Bhanuka and Raveen, for their unconditional support.

\section{References}

1. How Doctors Think. Jerome Groopman. ISBN 0-618-61003-0. Houghton Mifflin Company; 2007.

2. Thompson C, Dowding D. Clinical Decision Making and Judgement in Nursing. London Churchill Livingstone; 2002.

3. Why to read them and how to start reading them critically. Can Med Assoc J 1981;124: 555-8.

4. Tracy CS, Dantas GC, Moineddin R, Upshur REG. The nexus of evidence, context, and patient preferences in primary care. BMC Family Practice 2003; 4: 13. 
5. Saarni SI, Gylling HA. Evidence based medicine guidelines: a solution to rationing or politics disguised as science? Journal of Medical Ethics 2004; 30: 171-5.

6. NOF Clinician's guide to prevention and treatment of osteoporosis. National Osteoporosis Foundation. www.nof.org/professionals/clinical-guidelines/ (accessed last 25th July 2012).

7. Kanis JA, McCloskey EV, Johansson $\mathrm{H}$, Strom O, et al. Case findings for the management of osteoporosis with FRAX-assessment and intervention thresholds for the UK. Osteoporosis International 2008; 19(10): 1395-408.

8. Fundamentals of Evidence-Based Medicine. Prasad K. ISBN 81-7525-539-0. Meeta publishers, New Delhi; 2004.

9. Clinical Examination in Medicine. Jain AP, Goel A. ISBN 81-8191-031-1 PARAS publishing India; 2004.

10. Travers J, Marsh S, Caldwell B, et al. External validity of randomized controlled trials on COPD. Respiratory Medicine 2007; 101(6): 1313-20.

11. Travers J, Marsh S, Williams M, et al. External validity of randomized controlled trials in asthma: to whom do the results of the trials apply? Thorax 2007; 62: 219-23.

12. Comparison of coronary bypass surgery with angioplasty in patients with multivessel disease. The Bypass Angioplasty Revascularization Investigation (BARI) Investigators. NEJM 1996; 335(4): 217-25.

13. Assmann SF, Pocock SJ, Enos LE, Kasten LE. Subgroup analysis and other (mis)uses of baseline data in clinical trials. Lancet 2000; 355: 1064-69.

14. Bhandari M, Devereaux PJ, Patricia L, et al. Misuse of baseline comparison tests and subgroup analyses in surgical trials. Current Orthopedic Practice 2006; 247-51.

15. Jefferys DB, Leakey D, Lewis JA, Payne S, Rawlins MD. New active substances authorized in the UK between 1972 and 1994. Br J Clin Pharmacol 1998; 45: 151-6. 